\title{
Immunohistochemical analysis of Sonic hedgehog signalling in normal human urinary tract development
}

\author{
Dagan Jenkins, ${ }^{1,2}$ Paul J. D. Winyard ${ }^{1}$ and Adrian S. Woolf ${ }^{1}$ \\ ${ }^{1}$ Nephro-Urology, and ${ }^{2}$ Clinical and Molecular Genetics Units, UCL Institute of Child Health, UK
}

\begin{abstract}
Studies of mouse mutants have demonstrated that Sonic hedgehog $(\mathrm{SHH})$ signalling has a functional role in morphogenesis and differentiation at multiple sites within the forming urinary tract, and urinary tract malformations have been reported in humans with mutations that disrupt SHH signalling. However, there is only strikingly sparse and fragmentary information about the expression of $\mathrm{SHH}$ and associated signalling genes in normal human urinary tract development. We used immunohistochemistry to demonstrate that SHH protein was localised in distinct urinary tract epithelia in developing normal humans, in the urothelium of the nascent bladder and in kidney medullary collecting ducts. The expression patterns of the SHH-transducing proteins Patched (PTCH) and Smoothened (SMO) were consistent with long-range paracrine signalling associated with detrusor smooth muscle differentiation in the urogenital sinus. In the developing kidney, SHH and PTCH were expressed in epithelia of the collecting system between 16-26 weeks - surprisingly, SMO was not detected. Analysis of cell proliferation and Cyclin B1 immunohistochemistry at 26 weeks, as compared with a 28 week sample in which SHH expression was down-regulated, was consistent with the idea that SHH and PTCH might influence medullary collecting duct growth by regulating the subcellular localisation of Cyclin B1 independently of SMO. Collectively, these descriptive results generate new hypotheses regarding $\mathrm{SHH}$ signal transduction in human urinary tract development and help to explain the varied urinary tract malformation phenotypes noted in individuals with mutations in the $\mathrm{SHH}$ pathway.
\end{abstract}

Key words bladder; collecting duct; smooth muscle; urothelium; uroplakin.

\section{Introduction}

The hedgehog $(H H)$ gene was initially implicated in controlling segment polarity in fruit fly embryos; subsequently, the orthologous vertebrate Sonic hedgehog $(\mathrm{SHH})$ gene was identified and found to function as a secreted morphogen which, for example in mice, controlled cell fates in the neural tube and patterned limb buds (Ingham \& McMahon, 2001; Ingham \& Placzek, 2006). A large body of evidence has established a paradigm for the transduction of a $\mathrm{SHH}$ signal: cells that are responsive to $\mathrm{SHH}$ express the receptor Patched (PTCH), and binding of secreted SHH to PTCH relieves the normal repression of a second transmembrane protein Smoothened (SMO), the latter step mediated by pro-vitamin D3 secretion (Bijlsma et al. 2006). In vertebrates, this results in modulation of three intracellular proteins, the glioma-associated oncogenes (GLI1, GLI2 and GLI3) which variously act as transcriptional repressors and/or activators in target cells.

\section{Correspondence}

Dagan Jenkins, Nephro-Urology Unit, UCL Institute of Child Health, 30 Guilford Street, London, UK. T: 01865222 380;

E: dagan.jenkins@imm.ox.ac.uk

Accepted for publication 2 July 2007
While this paradigm has served as the basis for numerous investigations of $\mathrm{HH}$ signalling, new insights have demonstrated that $\mathrm{SHH}$ and $\mathrm{PTCH}$ can regulate cellular proliferation and apoptosis via mechanisms that, intuitively, bypass the requirement for SMO and the transcriptional response to morphogen. For example, PTCH can bind to Cyclin B1 and sequester it at the surface of cells, and binding of $\mathrm{SHH}$ to PTCH is associated with cell cycle progression by facilitating Cyclin B1 translocation to the nucleus (Barnes et al. 2001, 2005). Furthermore, caspase cleavage of the C-terminus of unbound PTCH uncovers a pro-apoptotic domain, and this effect is relieved by SHH binding (Thibert et al. 2003) - however, to date no evidence supporting the operation of these pathways in vivo has been generated.

In recent years, studies of embryonic mice have shown that $\mathrm{SHH}$ signalling has a functional role in morphogenesis and differentiation at multiple sites within the forming urinary tract (Mo et al. 2001; Yu et al. 2002; Haraguchi et al. 2007). Therefore, SHH signalling has been considered to play pleiotropic, complex, roles in urinary tract development. These basic science observations are made clinicallyrelevant by the fact that urinary tract malformations have been reported in humans with defined mutations of $\mathrm{SHH}$ and related signalling pathway genes: these are described in detail in the Discussion (Kelley \& Hennekam, 2000; 
Dubourg et al. 2004; Johnston et al. 2005). In the current study, we explored the expression of $\mathrm{SHH}$ and associated pathway molecules, PTCH and SMO, using immunohistochemistry of lower (i.e. urogenital sinus and bladder) and upper (i.e. kidney) urinary tract structures in human embryos and fetuses. In addition, because bone morphogenetic protein 4 (BMP4) has been implicated as a SHH-target molecule in regions of epithelial/mesenchymal interaction (Bitgood \& McMahon, 1995; Sukegawa et al. 2000; Yu et al. 2002), and because BMP4 itself is considered to be a pleiotropic effector of urinary tract morphogenesis (Miyazaki et al. 2000; Raatikainen-Ahokas et al. 2000; Lamm et al. 2001; Miyazaki et al. 2003), we also examined the expression of this protein in relation to $\mathrm{SHH}$ pathway proteins.

\section{Materials and methods}

\section{Human tissues}

Phenotypically normal human embryos from the first trimester at 4 weeks ( 2 embryos), and at 7 and 13 weeks (1 embryo each) were obtained from chemically induced terminations of pregnancy with informed consent. The embryonic tissues were obtained from the Wellcome Trust and Medical Research Council-funded Human Developmental Biology Resource at the UCL Institute of Child Health (Lindsay \& Copp, 2005). Later antenatal kidney samples (8 fetuses) from spontaneously miscarried fetuses were obtained from University College London Medical School, and were deemed to be normal by a trained pathologist. Use of this material was approved by the Joint University College London/University College Hospital Committee on the Ethics of Human Research.

Embryos were collected immediately after termination, fixed intact in cold 4\% paraformaldehyde/PBS ( $\mathrm{pH} 7.0$ ) overnight at $4{ }^{\circ} \mathrm{C}$ and processed for histology. Kidneys from normal human fetuses at 16, 18, 20, 25, 26, 28 (one each) and 23 ( 2 embryos) weeks' gestation, in the second trimester, comprised normal kidneys from spontaneous miscarriages. The fetus was stored at $4{ }^{\circ} \mathrm{C}$ until examination when organs were fixed in $10 \%$ formalin. All specimens were processed using routine laboratory procedures within $24 \mathrm{~h}$. Tissues were aged by Carnegie Staging (first-trimester samples) or by the time since the last period (second trimester), which was assumed to occur 2 weeks before conception. In addition, key histological features were used to stage tissues; for example, metanephroi at 7 weeks contain their first layer of glomeruli and nephrogenesis ceases after 36 weeks gestation (Woolf \& Jenkins, 2006).

\section{Antibodies}

A goat polyclonal anti-SHH antibody (N-19) raised against the $\mathrm{N}$-terminus of human $\mathrm{SHH}$ precursor (immunohistochemistry; 1:50), and goat polyclonal anti-
PTCH (C-20, raised against the C-terminal 20 amino acids of the human protein; 1:200), rabbit polyclonal anti-SMO $(\mathrm{H}-300$, raised against amino acid residues $488-787$ of the human protein; $1: 100$ ) and rabbit polyclonal anti-Cyclin B1 $(\mathrm{H}-20$, raised against the $\mathrm{C}$-terminal 20 amino acids of the human protein; 1:100) antibodies were purchased from Santa Cruz (Santa Cruz, CA, USA). These antibodies have been used previously and specificity has been confirmed by Western blotting (van den Brink et al. 2001, 2005; Munro et al. 2001; Oniscu et al. 2004). In particular, the anti-SHH antibody is specific to the $52 \mathrm{kDa} \mathrm{SHH}$ precursor protein, rather than active, secreted morphogen (19 kDa), and does not cross-react with Indian Hedgehog, as shown by Western blotting (van den Brink et al. 2001). Thus this anti-SHH antibody only labels cells that are the source of morphogen, rather than those cells expressing a $\mathrm{SHH}$ receptor; indeed, it has been shown that secreted $\mathrm{SHH}$ morphogen can only be detected by immunohistochemistry in tissues that have been fixed in such a way (not used in the current study) that preserves proteoglycans/glycosaminoglycans and proteins in soluble form (Gritli-Linde et al. 2001). A mouse monoclonal anti-BMP4 antibody (NCL-BMP4; 1:10) was purchased from Novocastra (Newcastle Upon Tyne, UK). A 'broad-spectrum' anti-Uroplakin (UP) antibody (AUM; 1:200; a gift from Prof. Tung-Tien Sun, New York University Medical Center) recognises all four UP proteins (Wu et al. 1994); the UPs are differentiation markers of urothelia (Romih et al. 2005). A monoclonal anti- $\alpha$ smooth muscle actin ( $\alpha$ SMA) antibody conjugated to alkaline phosphatase (A5691; 1:200) was purchased from Sigma (Steinheim, Germany), and a monoclonal anti-proliferating cell nuclear antigen (PCNA) antibody (1:200) was purchased from BD Pharmingen (Franklin Lakes, CA, USA). Negative controls included the replacement of primary antibody with preimmune mouse IgG for monoclonal antibodies, or with preimmune serum from the appropriate species for polyclonal antibodies; for SHH and Cyclin B1 immunohistochemistry, blocking peptides were available and competed efficiently with binding of the appropriate antibody. In all the negative control experiments, no signal was obtained for any of the antibodies under study.

\section{Immunohistochemistry}

Peroxidase-based immunohistochemistry using paraffinsections was performed as previously described (Jenkins et al. 2005). In some cases, following generation of a peroxidase-based signal using the AUM or SHH N-19 antibodies, a pink/red signal labeling $\alpha$ SMA-positive cells was generated by incubating with the alkaline phosphatase conjugated anti- $\alpha$ SMA antibody for $1 \mathrm{~h}$ at room temperature and, following three washes in PBS, a signal was generated using the Fast Red system (Sigma) according to the manufacturer's instructions. These slides were then mounted in Citifluor. 


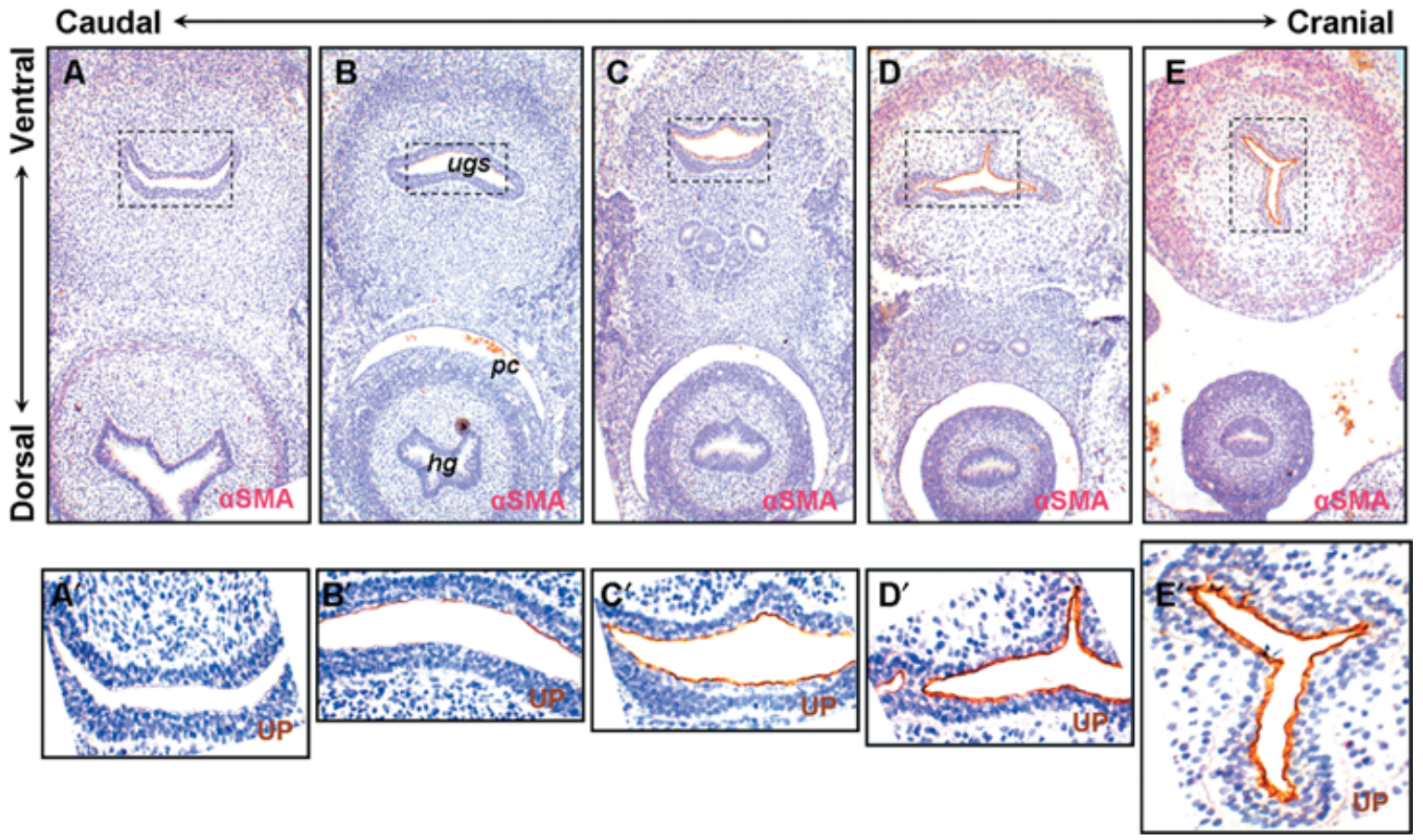

Fig. 1 Differentiation of the 7-week urogenital sinus. Expression of UP (brown signal) and $\alpha$ SMA (pink/red signal) in a series of transverse sections of the urogenital sinus (ugs) from most caudal (A) to most cranial (E) axial level, showing a graded intensity of UP and $\alpha$ SMA, in the urothelium and mesenchyme respectively, in the cranial-to-caudal axis. $A^{\prime}-E^{\prime}$, high-power views of the urothelium as indicated by the hashed boxes in A-E. hg, hindgut; ugs, urogenital sinus; $P c$, peritoneal cavity. $A-E, \times 12.5$ magnification; $A^{\prime}-E^{\prime}, \times 100$ magnification. Sections counterstained with hematoxylin.

\section{Results}

In the 4-week gestation embryo, SHH was immunolocalised in the epithelium of the urogenital sinus which was in continuity with the cloaca: at this stage there is no muscle around the nascent urothelium (data not shown). In a 7week gestation embryo, the epithelium of the urogenital sinus is seen to be distinct from that of the hindgut, with the interposition of the mesonephric and paramesonephric ducts and, more cranially, the peritoneal cavity, and has begun to undergo morphogenesis to produce a recognisable bladder outlet/proximal urethra and bladder dome, as shown in the caudal-to-cranial sequence depicted in Fig. 1. Immunohistochemical analysis of this series of sections showed that the expression of markers of detrusor smooth muscle ( $\alpha S M A$, the red signal in Fig. 1A-E) and urothelial (UP, the brown color in Fig. $1 A^{\prime}-E^{\prime}$ ) differentiation were progressively more intense in the cranial regions of the urogenital sinus. Furthermore, we noted that differentiating smooth muscle enveloped the whole dome of the bladder, and was more prominent ventrally on sections below (more caudal to) the dome. These results illustrate the harmonised differentiation of bladder epithelium and mesenchyme. Indeed, later, at 13 weeks, both UP and $\alpha$ SMA expression were additionally detected at more caudal axial levels and muscle bundles were clearly visible (Fig. 2A-E); collectively, the data presented in Figs 1 and 2 indicate that the most cranial portion of the urogenital sinus differentiates first, and that differentiation proceeds in a cranial-to-caudal sequence.

Having shown that different axial levels of the seven week urogenital sinus appeared to represent distinct stages of muscularisation, we were able to investigate the relationship between $\mathrm{SHH}$ expression and smooth muscle differentiation. $\mathrm{SHH}$ was immunolocalised in the human urogenital sinus urothelium (Fig. $3 A^{\prime}-E^{\prime}$ ), the onset of expression slightly 'preceding' (i.e. more caudal than) smooth muscle differentiation (compare Fig. $3 C^{\prime}, D$ ). Consistent with the regulation of differentiation by $\mathrm{SHH}$ signalling, PTCH, SMO and BMP4 were immunolocalised in a very similar pattern in the surrounding mesenchyme (Fig. 4), coinciding with the expression of $\alpha \mathrm{SMA}$ (see similar levels in Figs $1 \mathrm{E}$ and $3 \mathrm{E}$ ). Using the same methods we were unable to detect SHH, PTCH, SMO or BMP4 in sections of the 13 week bladder (data not shown). Taken together, these data are consistent with the idea that, as the urogenital sinus epithelium undergoes further differentiation, as evidenced by UP expression, it acts as a 'signalling-centre', releasing $\mathrm{SHH}$ to initiate and enhance detrusor smooth muscle differentiation.

Next we sought expression of SHH in the metanephros, the direct precursor of the adult kidney, between 4 and 28 weeks' gestation. At 4 weeks, no SHH, PTCH or SMO was detected in the ureteric bud; similarly, no signals for these proteins were visualised through to 13 weeks (data not shown). We first detected expression of SHH and PTCH at 


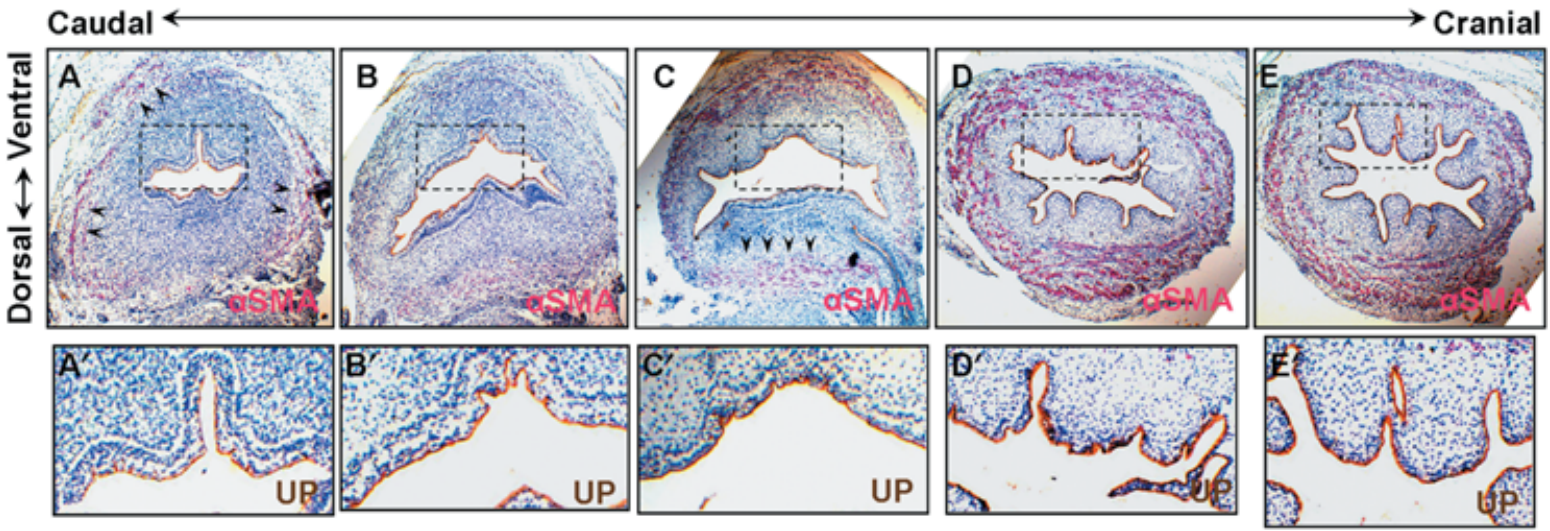

Fig. 2 Differentiation of the 13-week bladder. Expression of UP (brown signal) and $\alpha$ SMA (pink/red signal) in a series of transverse sections in the caudal to cranial axis of the 13 week bladder (A-E). $A^{\prime}-E^{\prime}$, high-power views of the urothelium as indicated by the hashed boxes in $A-E$. Note that both differentiation markers are now expressed at all axial levels, both ventrally and dorsally, and that $\alpha \mathrm{SMA}$ expression has resolved into muscle bundles. Arrowheads indicate $\alpha$ SMA expression in muscle bundles in the most caudal section in A, which corresponds to an axial level situated between that which is indicated in Fig. $1 B$ and $C$, and in the dorsal mesenchyme in $C$, which corresponds to an axial level equivalent to Fig. 1D. A-E, $\times 12.5$ magnification; $A^{\prime}-E^{\prime}, \times 100$ magnification. Sections counterstained with hematoxylin.

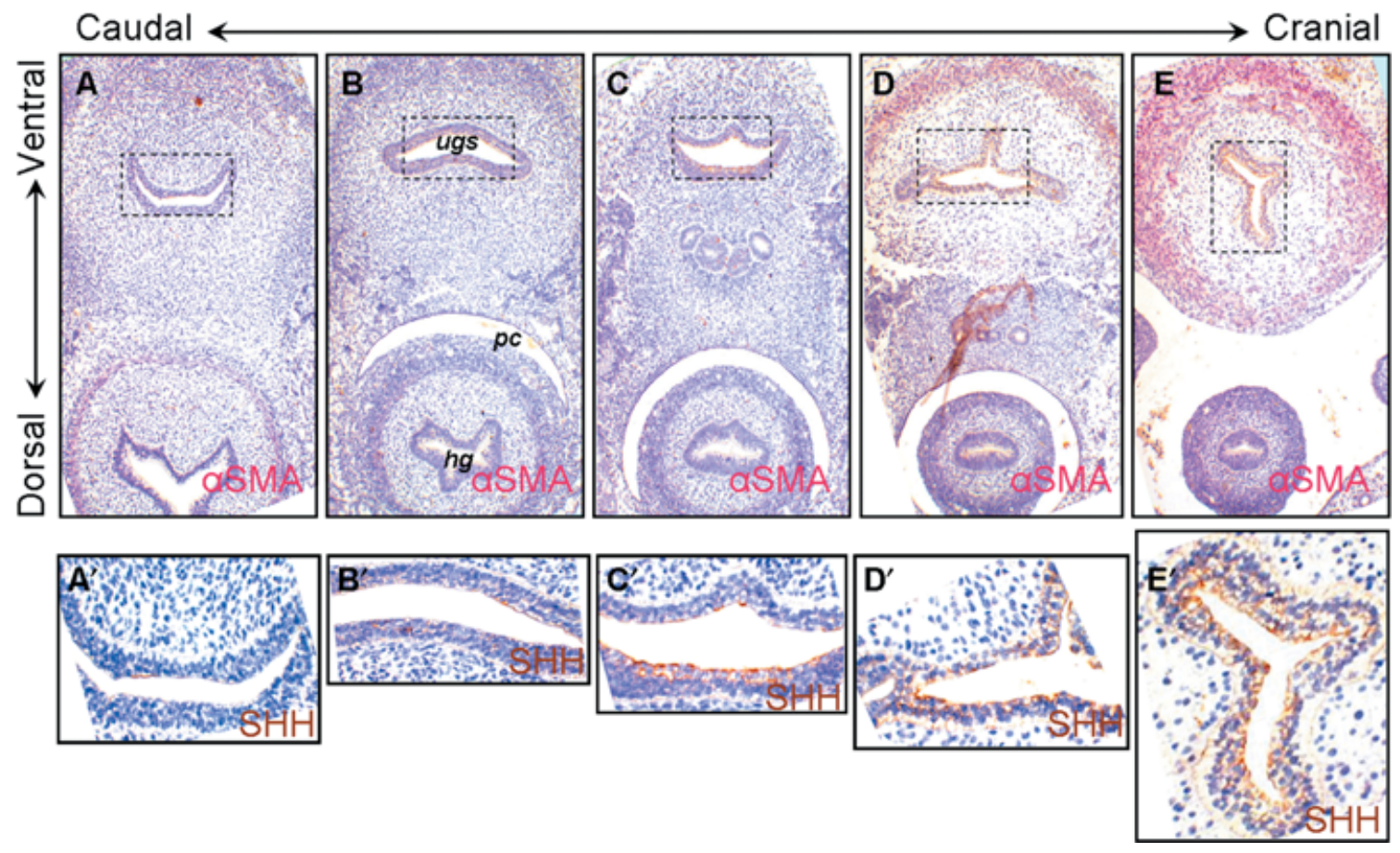

Fig. 3 Expression of Sonic hedgehog in the 7-week urogenital sinus. Expression of SHH (brown signal) and $\alpha$ SMA (pink/red signal) in a series of transverse sections of the urogenital sinus (ugs) from most caudal (A) to most cranial (E) axial level. $A^{\prime}-E^{\prime}$, high-power views of the urothelium as indicated by the hashed boxes in $\mathrm{A}-\mathrm{E}$. Note the graded intensity of $\mathrm{SHH}$ immunostaining of urothelium in the cranial to caudal axis, and the onset of its expression correlates with the onset of $\alpha \mathrm{SMA}$ expression. $\mathrm{hg}$, hindgut; ugs, urogenital sinus; $\mathrm{pc}$, peritoneal cavity. A-E, $\times 12.5$ magnification; $A^{\prime}-E^{\prime}, \times 100$ magnification. Sections counterstained with hematoxylin.

16 weeks (Fig. 5), when prominent signals were noted in renal pelvic epithelia and medullary collecting ducts, with continued expression through to 26 weeks (Fig. 6A,B). At these later gestations in the second trimester, we failed to immunodetect SHH or PTCH protein in the nephrogenic mesenchyme zone, and it was also absent in nephron precursors, forming in the outer cortex of the kidney
(Fig. 5F,G). At no stage could we immunodetect SMO within the developing kidney (Fig. $5 \mathrm{H}, \mathrm{I})$, while BMP4 was immunolocalised in glomerular parietal epithelia as well as cortical tubules at 13 weeks (Fig. $5 \mathrm{~K}$ ), consistent with studies in mouse embryonic kidneys (Dudley \& Robertson, 1997); these locations were distant from SHH-expressing medullary epithelia. Likewise, transcripts for the three 

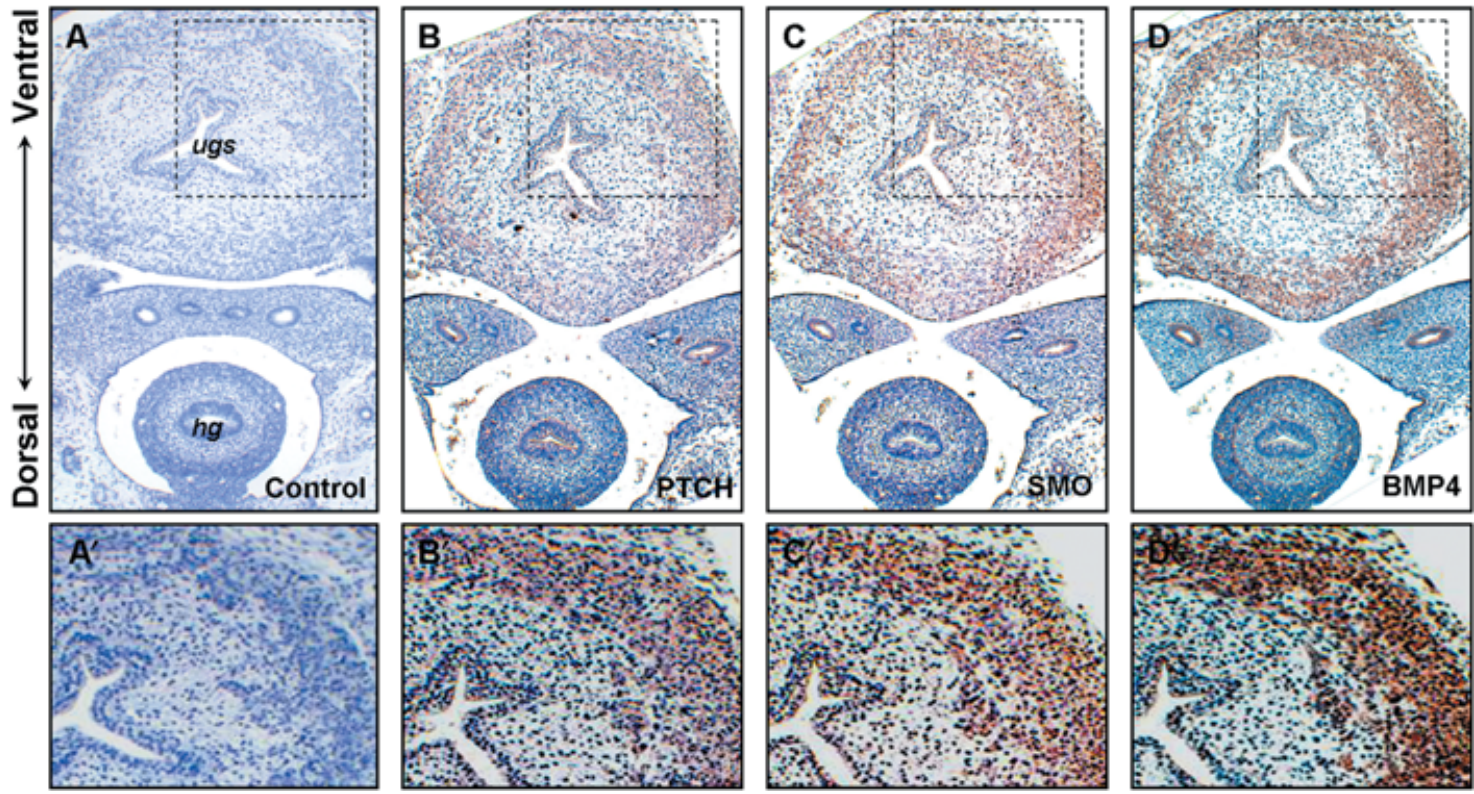

Fig. 4 Expression of SHH signal transduction molecules in the 7-week urogenital sinus. Immunolocalisation of PTCH (B), SMO (C) and BMP4 (D) in the peripheral mesenchyme of the 7-week urogenital sinus. (A) Negative control; antibody replaced with anti-rabbit lgG. Note the very close overlap of expression of all three proteins in adjacent sections, in condensing mesenchymal cells that express the differentiation marker $\alpha$ SMA (see Figs $1 \mathrm{E}$ and $3 E) . A-E, \times 12.5$ magnification; $A^{\prime}-E^{\prime}, \times 100$ magnification. Sections counterstained with hematoxylin.

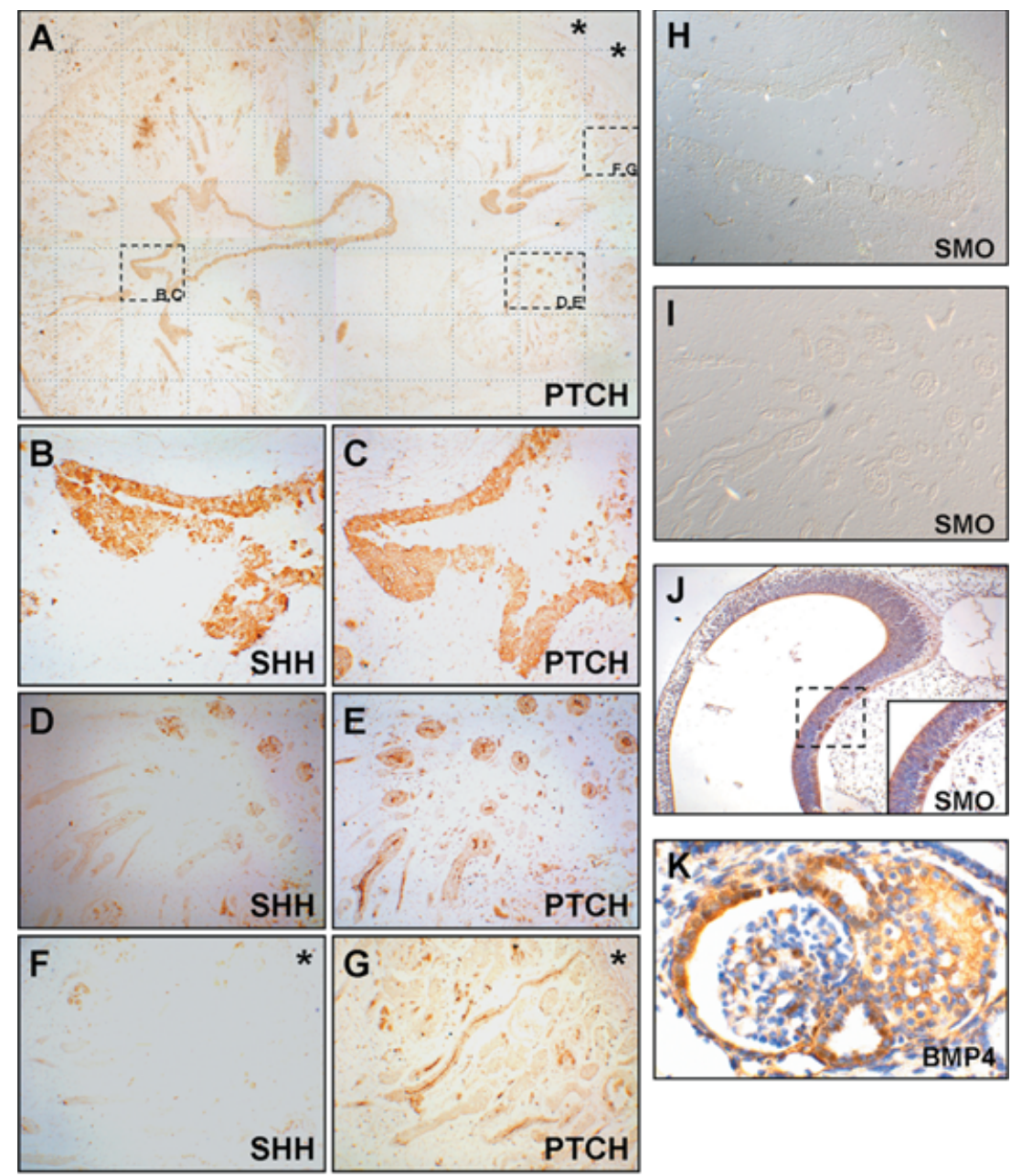

Fig. 5 Expression of SHH and PTCH in the 16-week kidney. (A) Low power view of PTCH expression in the 16-week kidney showing overall morphology. Inset dashed boxes refer to fields of view shown in B-G. B, D, F-expression of $\mathrm{SHH} ; \mathrm{C}, \mathrm{E}, \mathrm{G}-$ expression of PTCH. SHH and PTCH are expressed in the renal pelvis $(B, C)$, and medullary collecting ducts (D, E). PTCH (G), but not $\mathrm{SHH}(\mathrm{F})$, is also expressed in collecting ducts extending out to the capsule $\left(^{\star}\right)$ of the kidney. Neither SHH nor PTCH were detectable in the nephrogenic mesenchyme zone or in nephron precursors $(F, G)$. Note that PTCH is localised predominantly at the apical surface of collecting ducts. $\mathrm{H}, \mathrm{I}-$ fields of view equivalent to those shown in panels $B, C$ and $D, E$ respectively, showing no expression of SMO in the kidney; no immunoreactivity was detected for SMO in any of the kidney samples analysed between $16-28$ weeks. As a positive control for SMO immunohistochemistry, SMO protein was detected in the neuroepithelium of the developing forebrain (J, high magnification of the region indicated by the hashed box is inset) and ventricular mesenchyme of the heart (not shown) in a mid-sagittal section of a 10-week gestation embryo; SMO was also detected in the developing urogenital sinus at seven weeks (Fig. 4C, $C^{\prime}$ ). BMP4 was detected in glomerular parietal epithelia at 13 weeks' gestation (K).

A, $\times 12.5$ magnification; $B-J, \times 50$ magnification; $K, \times 100$. Sections in J, K counterstained with hematoxylin. 

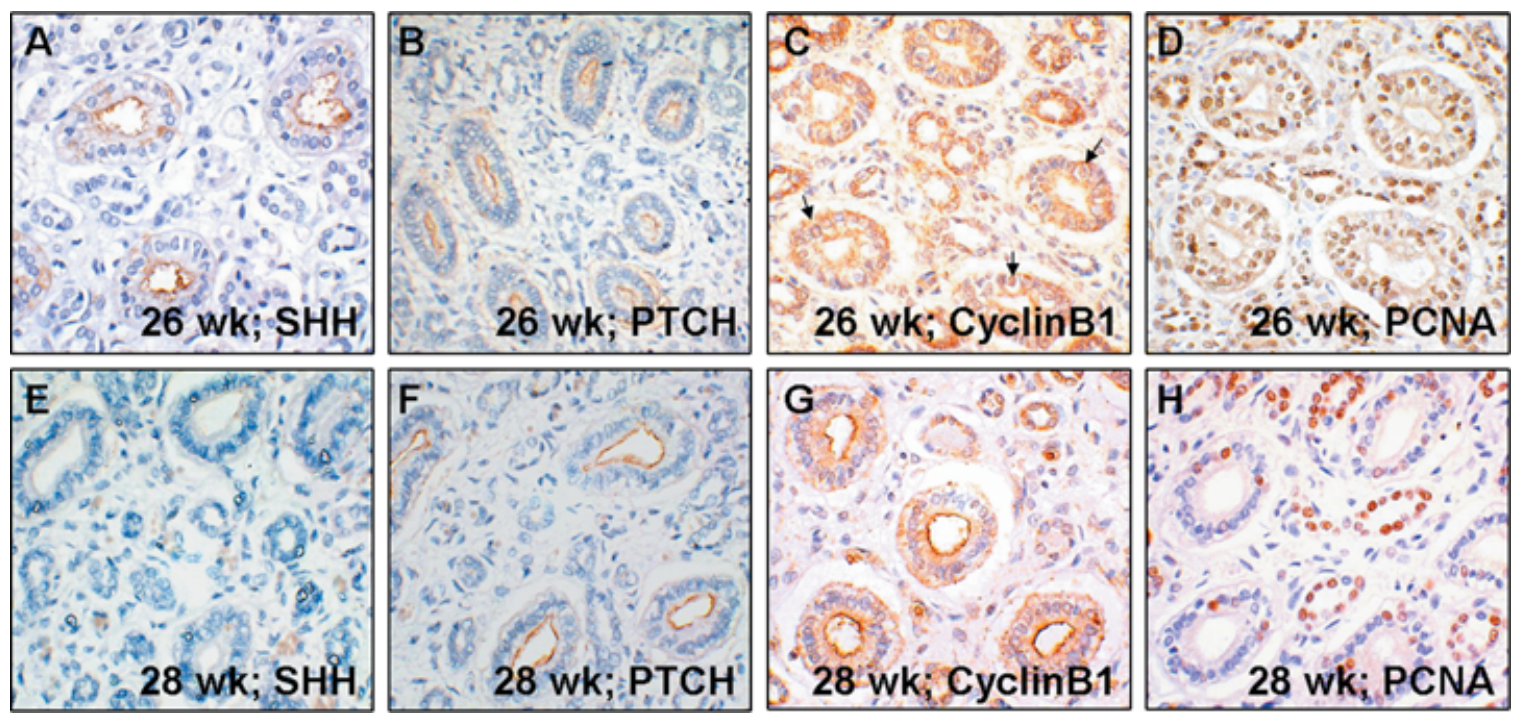

Fig. 6 Expression of SHH, PTCH, Cyclin B1 and PCNA in medullary collecting ducts of the 26- and 28-week kidneys. SHH is expressed in medullary collecting ducts at 26 weeks (A), but not at 28 weeks (E), and PTCH is expressed at both ages and is localised to the apical cell surface (B, F); both were absent from intervening interstitial cells and tubules of smaller diameter (presumably mainly representing loops of Henle). Absence of SHH at 28 weeks corresponds with a redistribution of Cyclin B1, from a diffuse cytoplasmic pattern (C) to the apical cell surface (G) in a similar location to that observed for PTCH (F). There is reduced proliferation of medullary epithelial cells at 28 weeks, compared to the 26 -week kidney, as evidenced by the proportion of PCNA-positive nuclei $(\mathrm{D}, \mathrm{H})$; note that this effect is limited to collecting duct cells, since no equivalent decrease in cell proliferation was observed in intervening epithelia. Arrows in C indicate occasional cell nuclei positive for Cyclin B1. Images at $\times 100$ magnification; sections counterstained with hematoxylin.

orthologous murine GLI genes have previously been detected only at metaneprhic locations (e.g. both GLI2 and GLI3 in the nephrogenic cortex) rather distant from the deep medullary source of SHH (Nakashima et al. 2002).

In the absence of SHH, PTCH can sequester Cyclin B1 at the cell surface and inhibit cell cycle progression (Barnes et al. 2001, 2005). While SHH expression was maintained in collecting ducts at 26 weeks (Fig. 6A), we found that it was down-regulated at 28 weeks (Fig. 6E), allowing us to investigate the effect of $\mathrm{SHH}$ on the subcellular distribution of Cyclin B1 in two similarly aged kidneys. Exploiting the polar distribution of PTCH at the apical surface of collecting duct cells (Fig. 6B,F), we assessed this possibility by analysing the subcellular distribution of Cyclin B1. In the 26-week sample, Cyclin B1 was immunodetected in all medullary tubules, in a diffuse cytoplasmic pattern (Fig. 6C), and within collecting ducts, $88 \%$ of all nuclei were positive for PCNA, a surrogate marker of proliferation (Fig. 6D). In the kidney at 28 weeks, in contrast, Cyclin B1 was also immunodetected in the medullary collecting ducts, but with accentuation of signal in the 'apical' zone of the epithelia (Fig. 6G), and the incidence of PCNA-positive cells $(28 \%)$ was less than in the 26 -week sample. In contrast to the apparent decrease in proliferation in medullary collecting ducts noted between the 26- and 28-week kidneys, there was no equivalent reduction in the incidence of PCNA-positive nuclei in the epithelia of the smaller, intervening tubules (92 versus $84 \%$ respectively).

\section{Discussion}

The phenotype of individuals with mutations of $S H H$, or other members of its signalling pathway, is often dominated by striking facial dysmorphology and associated anomalies of the brain and/or by tumour formation (Villavicencio et al. 2000; Nieuwenhuis \& Hui, 2004), and, as such, it is likely that examination of the urinary tract is not generally undertaken in a comprehensive manner (in life, by detailed radiology, or at autopsy). Nevertheless, urinary tract malformations have been documented. For example, Dubourg et al. (2004) reported that human SHH missense and nonsense mutations were, at times, associated with 'renal hypoplasia' and 'urogenital malformation'. Furthermore, GLI3 truncating mutations (which probably maintain the repressor but ablate the activator activity of this factor) occur in Pallister-Hall syndrome and these individuals can have urogenital sinus, absent unilateral kidney function, small kidneys and vesicoureteric reflux (Johnston et al. 2005). The Smith-Lemli-Opitz syndrome (SLOS) is caused by mutations that disrupt cholesterol metabolism, and this is thought to interfere with autoproteolysis of $\mathrm{SHH}$ into an active morphogen, and also results in the accumulation of pro-vitamin D3, a SMO antagonist (Guy, 2000; Kelley \& Hennekam, 2000; Cooper et al. 2003; Bijlsma et al. 2006): in SLOS, a range of urinary tract anomalies have been described including bladder, ureter and kidney hypoplasia, as well as ectopic and duplex kidneys. 


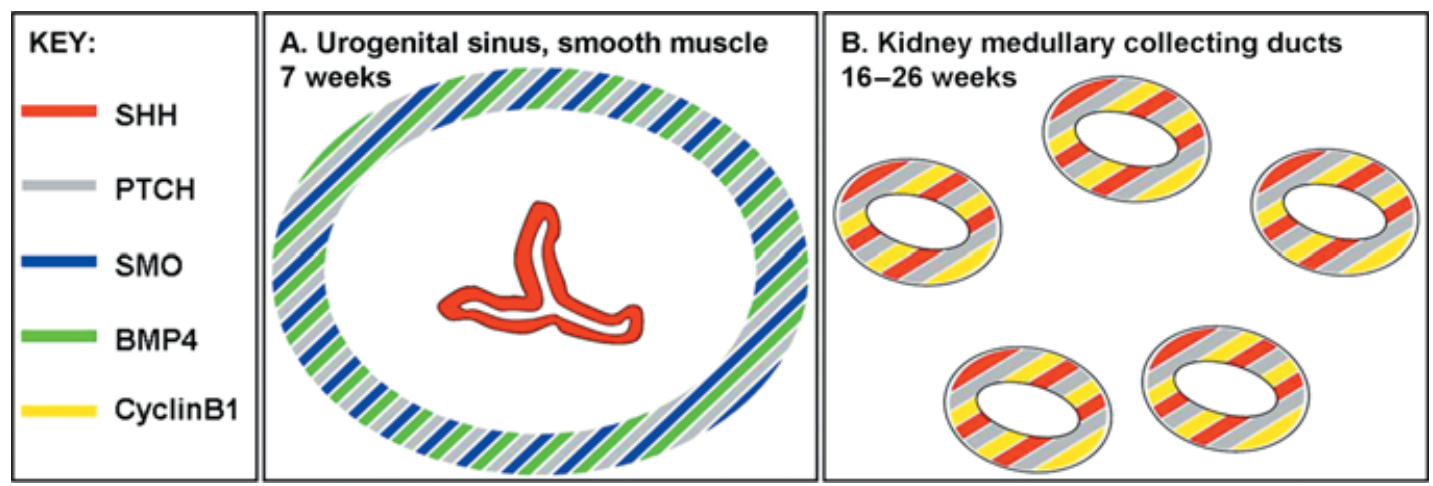

Fig. 7 Distinct molecular and cellular responses to SHH signalling in human upper and lower urinary tract development. Different colours in A and B illustrate the different structures in which various proteins were immunolocalised in this study, according to the key. A. SHH is expressed by urogenital sinus urothelium and signals to distant mesenchymal cells which express PTCH, SMO and BMP4, and which will form smooth muscle. These results are consistent with the regulation of smooth muscle differentiation by canonical SHH signalling. B. Epithelial cells of medullary kidney collecting ducts express SHH and PTCH, but not SMO. Descriptive results in vivo suggest that SHH signalling operates independently of SMO at short range in these cells to regulate cell proliferation according to the subcellular distribution of Cyclin B1.

Table 1 Summary of immunohistochemical analyses in normal human urinary tract tissues

\begin{tabular}{|c|c|c|c|c|c|}
\hline & & $\mathrm{SHH}$ & PTCH & SMO & BMP4 \\
\hline \multicolumn{6}{|l|}{ Lower urinary tract } \\
\hline \multicolumn{2}{|c|}{7 week cranial UGS - epithelium } & + & - & - & - \\
\hline \multicolumn{2}{|c|}{7 week cranial UGS - mesenchyme } & - & +nascent smooth muscle & $\begin{array}{l}\text { +nascent } \\
\text { smooth muscle }\end{array}$ & $\begin{array}{l}\text { +nascent } \\
\text { smooth muscle }\end{array}$ \\
\hline \multicolumn{2}{|c|}{13 week bladder - urothelium } & - & - & - & - \\
\hline \multicolumn{2}{|c|}{13 week bladder - smooth muscle } & - & - & - & - \\
\hline \multicolumn{6}{|l|}{ Kidney } \\
\hline \multirow[t]{2}{*}{4 week metanephros } & ureteric bud & - & - & - & - \\
\hline & mesenchyme & - & - & - & - \\
\hline \multirow[t]{3}{*}{ 7-13 week kidney } & pelvis & - & - & - & - \\
\hline & medulla & - & - & - & - \\
\hline & cortex & - & - & - & - \\
\hline \multirow[t]{3}{*}{ 16-26 week kidney } & pelvis & +urothelium & +urothelium & - & - \\
\hline & medulla & +collecting duct epithelia & +collecting duct epithelia & - & - \\
\hline & cortex & - & +collecting duct epithelia & - & +glomeruli \\
\hline \multirow[t]{3}{*}{28 week kidney } & pelvis & NP & NP & NP & NP \\
\hline & medulla & - & +collecting duct epithelia & - & - \\
\hline & cortex & - & +collecting duct epithelia & - & +glomeruli \\
\hline
\end{tabular}

UGS, urogenital sinus; NP, not performed; +, immunohistochemistry positive; -, immunohistochemistry negative.

We found immunoreactivity for the $\mathrm{SHH}$ precursor protein in distinct epithelia in the upper and lower urinary tract at specific stages of development, showing that these cells express the SHH gene (see Antibodies section of the Materials and Methods); these human observations are generally in accord with SHH mRNA expression as revealed by in situ hybridisation in epithelia of the cloaca, the bladder and ureter, and medullary collecting ducts in developing mouse embryos (Nakashima et al. 2002; Yu et al. 2002; Haraguchi et al. 2007). Within different parts of the developing human renal tract, although $\mathrm{SHH}$ protein localisation was always epithelial, there were some regional variations with respect to the detection and localisation of related signalling proteins, and these patterns (summarised in Fig. 7 and Table 1) are instructive as to the molecular and cellular processes regulated by $\mathrm{SHH}$.

In the nascent mouse urinary bladder, the urothelium is envisaged as a signalling centre which induces surrounding undifferentiated cells to form the detrusor (Haraguchi et al. 2007). Consistent with similar roles in human urogenital sinus differentiation, $\mathrm{SHH}$ was expressed when smooth muscle differentiation was taking place and, in terms of the cranial-to-caudal sequence of urogenital sinus differentiation that we demonstrated, its onset of expression preceded that of $\alpha \mathrm{SMA}$. We also showed for the first time that $\mathrm{SHH}$ only becomes expressed at the onset of urothelial 
differentiation, as evidenced by UP expression, offering insight into the regulation and timing of expression of this morphogen. Thus we conclude that defects in smooth muscle formation most likely account for the hypoplastic bladder phenotypes occasionally observed in patients with genetic disruption of SHH signalling (Kelley \& Hennekam, 2000; Dubourg et al. 2004; Johnston et al. 2005). Similar mechanisms may also underlie the range of ureteric anomalies that have been reported in patients with either Pallister-Hall or Smith-Lemli-Opitz syndromes (Kelley \& Hennekam, 2000; Johnston et al. 2005), consistent with the phenotype of mice carrying a deletion of $\mathrm{SHH}$ in ureteric urothelial cells (Yu et al. 2002). In the human ureteric tissues available to us (up to 13 weeks' gestation), the expression of neither SHH nor $\alpha$ SMA could be detected in the ureter (data not shown) and thus we are unable to draw further parallels between the mouse and human data.

In contrast to the documented effects of exposing progenitor cells of urinary tract smooth muscle to $\mathrm{SHH}$ (Yu et al. 2002; Haraguchi et al. 2007), very little is known about the cell biological role of $\mathrm{SHH}$ signalling during kidney development. We could not detect SHH protein at the onset of human metanephrogenesis, nor was it detected in the nephrogenic zone at later gestations: while we can not rule out the possibility that $\mathrm{SHH}$ protein is present at levels below our detection limit, the results make it unlikely that SHH itself is involved in either the initiation of the human metanephros or in the subsequent formation of nephrons which occurs in the nephrogenic zone of the renal cortex. In accord with our human data, mouse studies have failed to detect significant levels of SHH transcripts in the nephrogenic zone of the metanephros (Nakashima et al. 2002; Yu et al. 2002). Yu et al. (2002) noted prominent SHH transcripts in fetal medullary collecting ducts, with surrounding interstitial stromal cells expressing PTCH mRNA. We detected SHH in medullary collecting ducts, in the human fetal kidney, where immunohistochemical signals were found between 16 and 26 weeks of gestation. In distinction from the mouse data, however, PTCH was detected in the same epithelia rather than the interstitium; our immunolocalisation of this receptor is supported by the redistribution of Cyclin B1 to the apical surface of collecting duct cells of the 28week kidney in which the SHH signal was no longer detected (Fig. 6). We failed to detect SMO in these or other metanephric cells as assessed by immunohistochemistry. Although it is possible that biologically active levels of SMO were indeed present below our detection limits, our data raise the possibility that signalling operates independently of SMO in medullary collecting duct cells.

Kim et al. (2001) noted that SHH null mutant embryonic mice sometimes have a solitary kidney (presumably from failure to form the organ) and GL12/GLI3 compound null mutants have fused (horseshoe) kidneys; these pheno- types most likely arise secondarily to laterality and midline defects, rather than because of defective signalling within the metanephros itself, since the kidneys that do form in mice of both genotypes are located ectopically. Hu et al. (2005) reported that biochemical (cyclopamine)-mediated SMO inhibition perturbed nephrogenesis in metanephric organ culture, providing perhaps the most direct evidence that signalling by $\mathrm{SHH}$ (or perhaps a related ligand such as Indian or Desert hedgehog) requires SMO to enhance metanephric growth in mice; however, these experiments are potentially confounded by the use of cyclopamine at a dose that has been shown to border on a cytotoxic concentration (Taipale et al. 2000). The logical implication of our data in the kidney, that SHH signalling operates independently of SMO and presumably the downstream transcriptional effectors, GLI1-3, would support the contention that these kidney malformations arise secondarily to abnormal embryonic development external to the kidney itself. On the other hand, reports of small/hypoplastic, but normally positioned kidneys in patients with a heterozygous SHH mutation (Dubourg et al. 2004), and in mice homozygous for a $\mathrm{SHH}$ null mutation specifically in epithelia of the ureter and renal collecting system (Yu et al. 2002), would be consistent with the mitogenic role of $\mathrm{SHH}$ that we demonstrated in kidney collecting duct cells.

Our descriptive results with regard to gene expression in the medullary collecting ducts would be in accord with a model whereby SHH and PTCH (without the presence of SMO) regulate the proliferation of collecting duct cells according to the subcellular distribution of Cyclin B1, as outlined by Barnes et al. $(2001,2005)$. In the human Basal Cell Nevus syndrome (Villavicencio et al. 2000; Nieuwenhuis \& Hui, 2004), caused by mutations of PTCH, the phenotype is characterised by the occurrence of skin tumors such as basal nevae. Based on our current data about renal gene expression in humans, we would predict that such individuals might also harbor urinary tract defects including failure of renal growth and differentiation, and in this regard we note reports of absent kidney, renal cysts and Wilms tumor reported in individuals with this syndrome (Santis et al. 1983; Bacanli et al. 2005; Cajaiba et al. 2006). On the other hand, we failed to detect overt mutations upon sequencing exons 13 and 14 of $P T C H$, encoding an intracellular loop which interacts with Cyclin B1 (Barnes et al. 2001, 2005), in a group of humans with diverse urinary tract malformations including persistent cloaca, multicystic kidney and posterior urethral valves (Jenkins, 2006).

In summary, our novel data support the idea that $\mathrm{SHH}$ is a urinary tract morphogen, with multiple actions during development: these findings suggest that distinct molecular and cellular responses to SHH can account for the varied malformation phenotypes noted in the upper and lower urinary tracts of individuals with mutations in $\mathrm{SHH}$ and associated pathway genes. 


\section{Acknowledgements}

We thank the MRCNellcome Trust Human Developmental Biology Resource at the UCL Instiute of Child Health for the provision of first trimester embryonic tissues; Rosemary Scott at University College Hospital for the provision of antenatal kidney samples; Maria Bitner-Glindzicz for review of the manuscript; and Sally Feather and Aliyah Madhany for useful discussions. This work was funded by the Medical Research Council (D.J., A.S.W.), Kids Kidney Research (P.J.W.) and Kidney Research UK (P.J.W.).

\section{References}

Bacanli A, Ciftcioglu MA, Savas B, Alpsoy E (2005) Nevoid basal cell carcinoma syndrome associated with unilateral renal agenesis: acceleration of basal cell carcinomas following radiotherapy. $J$ Eur Acad Dermatol Venereol 19, 510-511.

Barnes EA, Heidtman KJ, Donoghue DJ (2005) Constitutive activation of the shh-ptc1 pathway by a patched 1 mutation indentified in BCC. Oncogene 24, 902-915.

Barnes EA, Kong M, Ollendorff V, Donoghue DJ (2001) Patched1 interacts with cyclin $\mathrm{B} 1$ to regulate cell cycle progression. $E M B O$ 20, 2214-2223.

Bijlsma MF, Spek CA, Zivkovic D, van de Water S, Rezaee F, Peppelenbosch MP (2006) Repression of smoothened by patched-dependent (pro-)vitamin D3 secretion. PLoS Biol 4, e232.

Bitgood MJ, McMahon AP (1995) Hedgehog and Bmp genes are expressed at many diverse sites of cell-cell interaction in mouse embryos. Dev Biol 172, 126-138.

Cajaiba MM, Bale AE, Alvarez-Franco M, McNamara J, ReyesMugica M (2006) Rhabdomyosarcoma, Wilms tumor, and deletion of the patched gene in Gorlin syndrome. Nat Clin Pract Oncol 3, $575-580$.

Cooper MK, Wassif CA, Krakowiak PA, et al. (2003) A defective response to Hedgehog signaling in disorders of cholesterol biosynthesis. Nature Genet 33, 508-513.

Dubourg C, Lazaro L, Pasquier L, et al. (2004) Molecular screening of SHH, ZIC2, SIX3 and TGIF genes in patients with features of holoprosencephaly spectrum: mutation review and genotypephenotype correlations. Hum Mutat 24, 43-51.

Dudley AT, Robertson EJ (1997) Overlapping expression domains of bone morphogenetic protein family members potentially account for limit tissue defects in BMP7 deficient embryos. Dev Dyn 208, 349-362.

Gritli-Linde A, Lewis P, McMahon AP, Linde A (2001) The whereabouts of a morphogen: direct evidence for short-and graded long-range activity of hedgehog signaling peptides. Dev Biol 236, 364-386.

Guy RK (2000) Inhibition of sonic hedgehog autoprocessing in cultured mammalian cells by sterol deprivation. Proc Natl Acad Sci USA 97, 7303-7312.

Haraguchi R, Motoyama J, Sasaki H, et al. (2007) Molecular analysis of coordinated bladder and urogenital organ formation by Hedgehog signaling. Development 34, 525-533.

Hu MC, Mo R, Bhella S, Wilson CW, Chuang PT, Hui CC, et al. (2005) GLI3-dependent transcriptional repression of Gli1, Gli2 and kidney patterning genes disrupts renal morphogenesis. Development 133, 569-578.

Ingham PW, McMahon AP (2001) Hedgehog signalling in animal development: paradigms and principles. Genes Dev 15, 30593087.
Ingham PW, Placzek M (2006) Orchestrating ontogenesis: variations on a theme by sonic hedgehog. Nat Rev Genet 7, 841-850.

Jenkins D (2006) The genetics of human renal tract malformations. PhD thesis, London: University of London, pp. 1-254.

Jenkins D, Bitner-Glindzicz M, Malcolm S, et al. (2005) De novo Uroplakin IIla heterozygous mutations cause human renal adysplasia leading to severe kidney failure. J Am Soc Nephro/ 16, 2141-2149.

Johnston JJ, Olivos-Glander I, Killoran C, et al. (2005) Molecular and clinical analyses of Grieg cephalopolysyndactyly and Pallister-Hall syndromes: robust phenotype prediction from the type and position of GLI3 mutations. Am J Hum Genet 76, 609622.

Kelley RI, Hennekam RC (2000) The Smith-Lemli-Opitz syndrome. J Med Genet 37, 321-335.

Kim J, Kim P, Hui CC (2001) The VACTERL association: lessons from the Sonic hedgehog pathway. Clin Genet 59, 306-315.

Lamm ML, Podlasek CA, Barnett DH, et al. (2001) Mesenchymal factor bone morphogenetic protein 4 restricts ductal budding and branching morphogenesis in the developing prostate. Dev Biol 232, 301-314.

Lindsay S, Copp AJ (2005) MRC-Wellcome Trust Human Developmental Biology Resource: enabling studies of human developmental gene expression. Trends Genet 21, 586-590.

Miyazaki Y, Oshima K, Fogo A, Ichikawa I (2003) Evidence that bone morphogenetic protein 4 has multiple biological functions during kidney and urinary tract development. Kidney Int 63, 835-844.

Miyazaki Y, Oshima K, Fogo A, Hogan BL, Ichikawa I (2000) Bone morphogenetic protein 4 regulates the budding site and elongation of the mouse ureter. J Clin Invest 105, 863-873.

Mo R, Kim JH, Zhang J, Chiang C, Hui CC, Kim PC (2001) Anorectal malformations caused by defects in sonic hedgehog signaling. Am J Pathol 159, 765-774.

Munro J, Steeghs K, Morrison V, Ireland H, Parkinson EK (2001) Human fibroblast replicative senescence cann occur in the absence of extensive cell division and short telomeres. Oncogene 20, 3541-3552.

Nakashima M, Tanese N, Ito M, et al. (2002) A novel gene, GliH1, with homology to the Gli zinc finger domain not required for mouse development. Mech Dev 119, 21-34.

Nieuwenhuis E, Hui CC (2004) Hedgehog signalling and congenital malformations. Clin Genet 67, 193-208.

Oniscu A, James RM, Morris RG, Bader S, Malcomson RD, Harrison DJ (2004) Expression of Sonic hedgehog pathway genes is altered in colonic neoplasia. J Pathol 203, 909-917.

Raatikainen-Ahokas A, Hytonen M, Tenhunen A, Sainio K, Sariola H (2000) BMP-4 affects the differentiation of metanephric mesenchyme and reveals an early anterior-posterior axis of the embryonic kidney. Dev Dyn 217, 146-158.

Romih R, Korosec P, de Mello W Jr, Jezernik K (2005) Differentiation of epithelial cells within the urinary tract. Cell Tissue Res 320, 259-268.

Santis HR, Nathanson NR, Bauer SB (1983) Nevoid basal cell carcinoma syndrome associated with renal cysts and hypertension. Oral Surg Oral Med Oral Pathol 55, 127-132.

Sukegawa A, Narita T, Kameda T, et al. (2000) KThe concentric structure of the developing gut is regulated by Sonic hedgehog derived from endodermal epithelium. Development 127, 19711980.

Taipale J, Chen JK, Cooper MK, et al. (2000) Effects of oncogenic mutations in Smoothened and Patched can be reversed by cyclopamine. Nature 406, 1005-1009. 
Thibert C, Teillet MA, Lapointe F, Mazelin L, Le Douarin NM, Mehlen P (2003) Inhibition of neuroepithelial patched-induced apoptosis by sonic hedgehog. Science 301, 843-846.

van den Brink GR, Bleuming SA, Hardwick JC, et al. (2005) Indian Hedgehog is an antagonist of Wnt signaling in colonic epithelial cell differentiation. Nat Genet 36, 277-282.

van den Brink GR, Hardwick JC, Tytgat GN, Brink MA, Ten Kate FJ, Van Deventer SJ, et al. (2001) Sonic hedgehog regulates gastric gland morphogenesis in man and mouse. Gastroenterology 121, 317-328.

Villavicencio EH, Walterhouse DO, lannaccone PM (2000) The sonic hedgehog-Patched-Gli pathway in human development and disease. Am J Hum Genet 67, 1047-1054.
Woolf AS, Jenkins D (2006) Chapter 2. Development of the kidney. In Heptinstall's Pathology of the Kidney, 6th edition (eds Jennette JC, Olson JL, Schwartz MM, Silva FG), pp. 71-95. Philadelphia-New York, USA: Lippincott-Raven.

Wu XR, Lin JH, Walz T, Haner M, Yu J, Aebi U, Sun TT (1994) Mammalian uroplakins. A group of highly conserved urothelial differentiation-related membrane proteins. J Biol Chem 269, 13716-13724.

Yu J, Carroll TJ, McMahon AP (2002) Sonic hedgehog regulates proliferation and differentiation of mesenchymal cells in the mouse metanephric kidney. Development 129, 5301-5312. 